\title{
Field Evaluation of an Oviposition Deterrent for Management of Spotted-Wing Drosophila, Drosophila suzukii, and Potential Nontarget Effects
}

\author{
Anna K. Wallingford, ${ }^{1,2}$ Heather L. Connelly, ${ }^{1}$ Gabrielle Dore Brind'Amour, ${ }^{1}$ \\ Matthew T. Boucher, ${ }^{1}$ Agenor Mafra-Neto, ${ }^{3}$ and Greg M. Loeb ${ }^{1}$ \\ ${ }^{1}$ Cornell University, New York Agricultural Experiment Station, 630 W. North St., Geneva, NY 14456 (akw52@cornell.edu; \\ hlc66@cornell.edu; gb222@cornell.edu; mtb245@cornell.edu; gme1@cornell.edu), ${ }^{2}$ Corresponding author, e-mail: \\ annawllngfrd@gmail.com, and ${ }^{3}$ ISCA Technologies, Inc., 1230 Spring St., Riverside, CA 92507 (president@iscatech.com)
}

Received 30 March 2016; Accepted 5 May 2016

\begin{abstract}
Spotted-wing drosophila, Drosophila suzukii Matsumura (Diptera: Drosophilidae), is a polyphagous, invasive pest of small fruits. Current management relies heavily on chemical insecticides, and an effective oviposition deterrent could contribute to alternative management approaches that reduce the need for these chemical insecticides. A novel deployment method for repelling Drosophila suzukii, thereby reducing $D$. suzukii oviposition in fall-bearing red raspberry, was evaluated in the field. Infestations occurring within $4 \mathrm{~d}$ after deployment were significantly lower in 2-m-long plots (Rubus idaeus 'Caroline') treated with the repellent (20\% 1-octen-3-ol in specialized pheromone and lure application technology [SPLAT]) compared to control plots (blank SPLAT). Repellent-treated plots had roughly 28.8 and $49.5 \%$ fewer offspring reared per gram of fruit than control plots in two experiments, respectively. Nontarget effects were also evaluated in 2-m plot experiments as well as 5- by 5$\mathrm{m}$ plot experiments. There were no differences in the number of parasitic hymenoptera trapped on yellow sticky cards hung in repellent compared to control plots. While there were no differences in the number of visits to raspberry flowers observed by honey bees in repellent versus control plots, the number of visits by bumble bees was greater in repellent plots compared to control plots. Challenges regarding evaporation rates and potential uses for repellents in an integrated pest management program for the control of $D$. suzukii are discussed.
\end{abstract}

Key words: 1-octen-3-ol, repellent, parasitic hymenoptera, pollinator

Spotted-wing drosophila, Drosophila suzukii Matsumura (Diptera: Drosophilidae), is a polyphagous, invasive pest of small fruits and cherries, which incurs serious economic losses in many fruit-growing regions of the world (Walsh et al. 2011, Asplen et al. 2015). Unlike most other drosophilids that reproduce in overripe or decomposing fruit, the female of this species possesses a heavily sclerotized, serrated ovipositor that allows her to oviposit into ripening fruit and the resulting larvae render the fruit unmarketable (Atallah et al. 2014). Current management relies on an unsustainable schedule of foliar insecticide applications (Beers et al. 2011, Van Timmerren and Isaacs 2013, Diepenbrock et al. 2016). An effective oviposition deterrent could contribute to alternative management approaches that may reduce the need for these chemical insecticides.

Repellent odors have been identified that induce aversive behaviors in D. suzukii (Pham and Ray 2015, Wallingford et al. 2015, Renkema et al. 2016). Of these odors, octenol (1-octen-3-ol) has been demonstrated to reduce $D$. suzukii infestations in cultivated red raspberry in the field (Wallingford et al. 2015). While repellents are commonly used to protect mammalian hosts from blood-feeding arthropods (Lupi et al. 2013), this approach is rare in agricultural settings. An efficient deployment strategy is needed in order to maintain a high concentration of a volatile compound over days or weeks rather than the hours of protection often reported in mammalian systems.

Raspberry (Rubus idaeus L.) requires insect pollination for optimal yield and fruit quality. An aggregate fruit, or polydrupe, forms from the fertilization of many ovules within the same flower. Despite being self-fertile, insect pollination is necessary to ensure full fertilization and produce commercial-quality raspberries (Crane and Walker 1984). Raspberry flowering and fruit ripening occur at the same time within plantings, so crop protection is necessary while pollinators are visiting flowers. Regular use of broad-spectrum foliar insecticides is disruptive to pollinators as well as natural enemy complexes (Pimentel 1995). Avoidance behavior of beneficial insects, like pollinators, predators, and parasitic wasps, due to the presence of a repellent odor would detract from the value of a repellent as an alternative to foliar insecticides. 
Here we evaluate a novel deployment method for repelling $D$. suzukii and reducing oviposition in fall-bearing red raspberry. We also investigated potential nontarget effects of deploying high concentrations of a repellent odor in the field by conducting observations of pollinator visitation and by monitoring parasitic wasp densities in treated and untreated areas.

\section{Materials and Methods}

\section{Greenhouse Efficacy Trials}

In order to estimate an effective concentration of volatile octenol for deterring D. suzukii oviposition, caged, no-choice tests were conducted under greenhouse conditions $\left(22 \pm 1^{\circ} \mathrm{C}, 40-60 \%\right.$ relative humidity, and a photoperiod of 16:8 [L:D] h). Experiments were conducted in sleeve cages (90 by 45 by $45 \mathrm{~cm}$; BioQuip, Rancho Dominguez, CA) holding 50 colony-raised D. suzukii $(7 \mathrm{~d}$ old, 50:50 male:female) and four store-bought organic raspberry fruit sitting on an open polystyrene Petri dish (100-mm circumference). The fruit were arranged in a circle around a wick $(1$ by $1 \mathrm{~cm}$; Absorbal surgical cellulose wadding, Wheat Ridge, CO) sitting at the center of the dish, fruit receptacle side down so that flies could not hide inside. Treatments $(100 \mu \mathrm{l})$ were applied to the wick: 1 , 10 , or $50 \%$ octenol ( $\geq 98 \%$ 1-octen-3-ol; Sigma-Aldrich, St Louis, $\mathrm{MO})$ or solvent only (mineral oil, Sigma-Aldrich). Flies were allowed to oviposit for $4 \mathrm{~h}$, after which fruit were examined under a dissecting scope for eggs. Each treatment was replicated in eight cages and repeated on two separate dates $(n=16)$. The weight of each wick or stimulus was recorded before and after each experiment and the difference in weight was used to estimate hourly evaporative loss. Previous experiments found that weight loss using this method was linear for all concentrations for at least $6 \mathrm{~h}$ after application.

\section{Field Sites}

All field experiments were conducted in insecticide-free, primocane fruiting (fall-bearing) red raspberry plantings. Research farm sites A and B were neighboring plantings of Rubus idaeus 'Caroline' in Geneva, NY. The research plantings were approximately the same size ( 12 by $40 \mathrm{~m}$ ). Site A was established in the spring of 2015 while site B was planted in 2013. Although site A was a newly established planting, the majority of plants flowered and produced fruit by August and continued producing new fruit through October. The commercial farm site was located in Trumansburg, NY, also growing primocane fruiting $R$. idaeus ( 16 by $100 \mathrm{~m}$ ).

\section{Field Efficacy Trial}

A novel deployment method was evaluated for the management of D. suzukii in fall-bearing red raspberry at research farm site A in August 2015. The repellent was an experimental formulation containing $20 \%$ octenol ( $\geq 98 \%$ 1-octen-3-ol; Sigma-Aldrich) incorporated into specialized pheromone and lure application technology (SPLAT), a proprietary slow release matrix from ISCA Technologies to formulate semiochemical blends, among other active ingredients. For each of two experiments, 16 plots were flagged ( $2 \mathrm{~m}$ long) and any ripe fruit was removed, leaving behind green fruit, which would become ripe during the following $4-7 \mathrm{~d}$. For each plot, a coin flip determined assignment of control (blank) or repellent SPLAT treatments. Dolops of SPLAT were deployed in paper hangtags hooked around canes and stapled in place (2-3 g SPLAT per hangtag). In each plot, 10 hangtags were evenly spaced at fruit height within the plant canopy $(4-6 \mathrm{~g} / \mathrm{m})$. Ripe, market quality fruit were harvested at
4 and $7 \mathrm{~d}$ after SPLAT was deployed and returned to the lab where fruit was weighed and transferred to rearing containers (0.5-liter plastic deli cups with mesh fabric bottoms nested in 1-liter plastic deli cups and covered with mesh fabric to allow ventilation; Wallingford et al. 2015). Emerging adults were identified to species using wing and genital morphology, and the total number of D. suzukii offspring was tabulated after $14 \mathrm{~d}$ in rearing conditions $\left(22 \pm 2^{\circ} \mathrm{C}\right)$.

Two baited traps were deployed to measure adult activity for the duration of each experiment. Traps were $16 \mathrm{oz}$. red solo cups and lids (Dart Container Corp., Mason, MI) with 40 entry holes $(3.175 \mathrm{~mm})$, holding a fermenting wheat-bait $(17.25 \mathrm{~g}$ whole wheat flour, $25 \mathrm{ml}$ distilled water, $2 \mathrm{~g}$ sucrose, $1 \mathrm{ml}$ apple cider vinegar, and $0.325 \mathrm{~g}$ baker's yeast [ACH Food Companies Inc., Memphis, $\mathrm{TN}]$ ) over a drowning solution of apple cider vinegar (5\% acetic acid), ethanol $(10 \%)$, and a drop of odor-free dish detergent (Seventh Generation Inc, Burlington, VT). Trap contents were drained at the end of each experiment and held in ethanol $(70 \%)$ until the number of adult D. suzukii were counted under a dissecting microscope.

\section{Hangtag Evaporation Rates}

The amount of repellent evaporating from paper hangtags during field experiments was estimated in the laboratory using a weight loss method. Hangtags containing $2-3 \mathrm{~g}$ of SPLAT were aged in a fume hood (face velocity $=0.38 \mathrm{~m} / \mathrm{s}$ ) for $0,1,2,4,5,6$, or $7 \mathrm{~d}$. After aging, the weight of each hangtag was measured hourly for $4 \mathrm{~h}$ to estimate hourly evaporation rates.

\section{Pollinator Surveys}

Timed observations of naturally occurring pollinator visitation were conducted in the first two days of SPLAT deployment in three experiments at the commercial farm site and research sites A and B, August-September 2015. At each site, three interior rows were divided into 5 - by $5-\mathrm{m}$ plots. At the commercial farm site, eight plots were flagged with 3-m buffers between each plot. A coin flip was used to determine control or treatment assignments to plots in each of the four blocks. A similar protocol was carried out at research sites $\mathrm{A}$ and $\mathrm{B}$, where three interior rows were divided into eight and six plots, respectively. At research site A there were no buffers between plots while there were 1-m buffers between plots at research site B. Control or repellent SPLAT hangtags were deployed as previously described, but at a rate of 40 hangtags per plot $(5-8 \mathrm{~g} / \mathrm{m})$.

For each of the three experiments, observations were made on the day of deployment and again the following day (10AM-1PM). At the commercial farm site and at research site B, three observers counted the number of visits to raspberry flowers in each $5-\mathrm{m}$ row during a 3-min period for each plot, so that all three rows of the plot were being observed concurrently. This was repeated for each plot three times so that each row was observed once by each observer. A similar protocol was repeated at research site A with two observers and two observations of two rows in each plot.

\section{Parasitic Wasp Densities}

We also assessed nontarget effects of the repellent by sampling using yellow sticky traps in control and repellent plots in each of the 2-m plot experiments, the commercial site, and the 5 - by 5 -m plot experiment in research farm site A, as previously described. One yellow sticky card ( 7.6 by $12.7 \mathrm{~cm}$; Olson, Medina, $\mathrm{OH}$ ) was fixed with binder clips to a bamboo stakes in the center of each of the 2-m plot 
efficacy trials. In 5- by 5-m plot experiments, two sticky cards per plot were clipped to the canes of plants. Cards were removed $7 \mathrm{~d}$ after repellent deployment, wrapped in clear plastic wrap, and returned to the lab. Cards were held in a freezer $\left(-20^{\circ} \mathrm{C}\right)$ until they could be inspected under a dissecting scope. The total number of parasitic hymenoptera was recorded for each card, including members of Chrysidoidea, Cynipoidea, Chalcidoidea, Platygastroidea, Ceraphronoidea, Prototrupoidea, and Ichneumonoidea (Lee 2009).

\section{Statistical Analysis}

All analyses were carried out in JMP (SAS Institute, Cary, NC; $\alpha=0.05$ ). For greenhouse efficacy trials, a mixed-model analysis was carried out to determine the fixed effect of treatment (solvent, 1,10 , or $50 \%$ octenol) on infestation, considering experimental day as a random effect, followed by Tukey's HSD means separation. For field efficacy trials, a one-tailed Student's t-test was carried out to compare yield and infestation of fruit harvested from control and repellent plots. For pollinator surveys, the number of visits by honey bees, bumble bees, and other hymenoptera was averaged for each concurrent observation and a mixed-model analysis was carried out to determine the fixed effect of treatment (control, repellent) on number of visits, considering experiment (commercial site, research site A, site B) as a random effect, followed by Tukey's HSD means separation. A Student's t-test was used to compare number of parasitic hymenoptera trapped on sticky cards over $7 \mathrm{~d}$ in repellent versus control plots for each of the four experiments $(2 \mathrm{~m}$ experiment 1 and 2, commercial site, research site A). To characterize hourly evaporation rates of $0,1,2,4,5,6$, or 7 old SPLAT hangtags, the best fit line was determined from the regression of hourly weight loss over time for each age.

Table 1. Infestation from greenhouse efficacy trials, mean ( \pm SEM) eggs observed in raspberry fruit caged with 50, 7-d-old colonyraised $D$. suzukii for $4 \mathrm{~h}$, protected by varying concentrations of 1-octen-3-ol in mineral oil (v/v)

\begin{tabular}{ll}
\hline Treatment & Eggs/g fruit \\
\hline Solvent & $2.43 \pm 0.34 \mathrm{a}$ \\
$1 \%$-octen-3-ol & $1.81 \pm 0.28 \mathrm{ab}$ \\
$10 \% 1$-octen-3-ol & $1.61 \pm 0.22 \mathrm{ab}$ \\
$50 \%$-octen-3-ol & $1.22 \pm 0.19 \mathrm{~b}$
\end{tabular}

Values followed by the same letter are not different according to Tukey's HSD.

\section{Results}

In greenhouse efficacy tests, experiments using $50 \%$ octenol resulted in lower infestations than control experiments $(F=4.22, \mathrm{df}=3,63$, $P=0.0092$; Table 1). From the best fit line for evaporation rate $(\mathrm{mg} / \mathrm{h})$ versus infestation, we estimated a target deployment rate of at least $10 \mathrm{mg} / \mathrm{h}$ for repellency (Suppl. Fig. 1 [online only]).

In field efficacy trials, D. suzukii infestations $4 \mathrm{~d}$ after deployment were lower in repellent-protected fruit compared to controls for both experiments $(t=2.14, \mathrm{df}=1,15, P=0.0337$ and $t=1.85$, $\mathrm{df}=1,15, P=0.0441$, respectively; Table 2). Repellent-treated plots had roughly 28.8 and $49.5 \%$ fewer offspring reared per gram of fruit than control plots in two experiments, respectively. Heavy rains occurred during the following three days of the first experiment (which likely impaired insect flight) and no offspring were reared from control or treatment fruit, so no difference in infestation could be evaluated. There was no difference in infestation from fruit harvested $7 \mathrm{~d}$ after deployment in the second efficacy experiment (Table 2).

Weight loss over time was linear for control hangtags that were 0 or $1 \mathrm{~d}$ old and repellent hangtags that were $0,1,2,3,4$, or $5 \mathrm{~d}$ old. The mean weight lost from hangtags fell below the $10 \mathrm{mg} / \mathrm{h}$ target after $1 \mathrm{~d}$ of aging and decreased steadily (Fig. 1). Evaporation rates from hangtags that were 6 or $7 \mathrm{~d}$ old could not be estimated using this method, as there was no detectable decrease over a 4 -h period.

There was no difference between control and repellent plots in the number of visits observed by honey bees or other hymenoptera; however, more visits by bumble bees were observed in repellent plots than in control plots $(F=7.895, \mathrm{df}=1,57, P=0.0059$; Fig. 2).

The number of parasitic hymenopterans captured on sticky cards was not different in repellent versus control plots at any site. The mean $( \pm$ SEM) number of parasitic wasps captured was $8.7 \pm 0.9$, $15.8 \pm 1.7,17.8 \pm 2.1$, and $22.4 \pm 2.0$ for $2-\mathrm{m}$ plot experiments 1 and 2 , the commercial site, and the 5 - by $5-\mathrm{m}$ plot experiment at research site A, respectively.

\section{Discussion}

The reduced infestations we observed in the greenhouse efficacy trials and in the first $4 \mathrm{~d}$ of the field efficacy trials is noteworthy (Tables 1 and 2). While not a standalone management tool at this stage, a repellent would be compatible with regular insecticide rotations and could contribute to an integrated pest management program for control of D. suzukii with improvements to deployment

Table 2. Yield and infestation from field efficacy trials conducted in August and September 2015, mean ( \pm SEM) weight of fruit harvested from 2 -m plots and number of spotted-wing drosophila reared per gram of fruit

\begin{tabular}{|c|c|c|c|c|}
\hline \multicolumn{2}{|c|}{ Day 4} & \multicolumn{2}{|c|}{ Day 7} & \multirow{2}{*}{$\begin{array}{c}\text { Adult capture } \\
\text { (spotted-wing } \\
\text { drosophila/trap/d) }\end{array}$} \\
\hline Yield (g) & $\begin{array}{c}\text { Infestation } \\
\text { (offspring/g fruit) }\end{array}$ & Yield (g) & $\begin{array}{c}\text { Infestation } \\
\text { (offspring/g fruit) }\end{array}$ & \\
\hline
\end{tabular}

Experiment 1

Control

$42.4 \pm 6.7$

$0.45 \pm 0.15$

$25.2 \pm 3.7$

2.7

Repellent

$43.8 \pm 5.1$

$0.13 \pm 0.02 *$

$21.8 \pm 4.2$

0

0

Control

$40.8 \pm 3.8$

$1.09 \pm 0.24$

$28.1 \pm 5.9$

$0.92 \pm 0.20$

$30.5 \pm 3.7$

$0.97 \pm 0.39$

$47.2 \pm 4.4$

\footnotetext{
(1)
}

Mean number of female spotted-wing drosophila captured per trap per day in baited traps over each 7-d experiment.

* Asterisk indicates value is significantly lower than the control according to a one-tailed Student's $t$-test. 


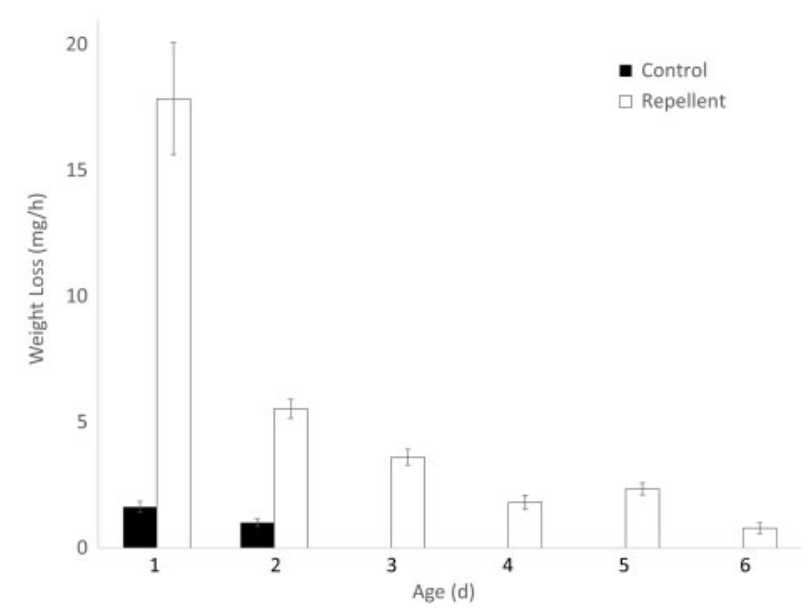

Fig. 1. Mean $( \pm$ SEM) hourly weight loss from hangtags after aging in a hood for $0-5 d$.

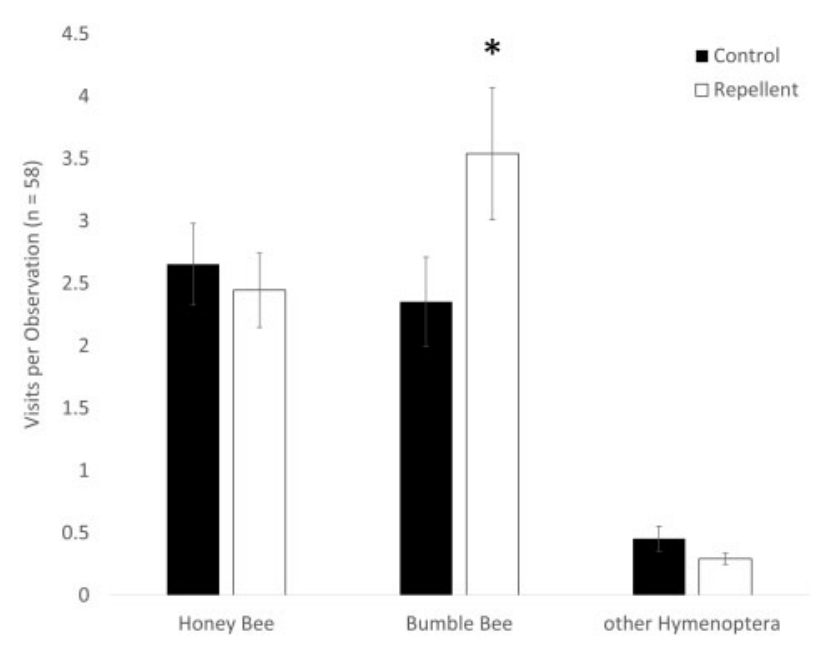

Fig. 2. Pollinator visitation observed in three experiments conducted in August-September 2015, mean ( \pm SEM) number of visits to raspberry flowers by honey bees, bumble bees, or other hymenopterans during a 3-min observation. Asterisk indicates significant difference according to mixed-model analysis.

(Haye et al. 2016). Complete control of D. suzukii populations is challenging, even with weekly application of foliar insecticides (Diepenbrock et al. 2016). High-pressure populations occur late in the season and are particularly challenging in fields neighboring alternate host species (Lee et al. 2015, Klick et al. 2016). There is potential for repellents to decrease pest pressure, thereby improving foliar insecticide program efficacy, or to extend intervals between foliar applications.

A repellent could also be combined with attractive odors to create a push-pull strategy that improves shortcomings of using either repellents or attractants on their own to alter insect behavior (Cook et al. 2007). The attractive odors that are meant to "pull" pests away from a target, where they are trapped in a mass-trapping device or killed with an insecticide, often create issues with recruitment. A very attractive stimulus will concentrate pest insects to areas around a mass-trapping device and therefore increase infestation within proximity to that point source (Hampton et al. 2014). Protecting this fruit with a repellent may counteract the problems associated with recruitment. Additionally, the success of a "push" strategy like the use of repellents may benefit from the addition of attracticidal baits. Assuming that the repellent acts to reduce oviposition by diverting oviposition site-searching behaviors to areas where the repellent is in lower concentrations, fruit that is farther away from a repellent point source would not be as well protected. A very attractive stimulus, like an attracticidal bait, could offer a better alternative than nearby fruit.

While the underlying mechanisms for oviposition deterrence due to the presence of the repellent are unclear and warrant further study, the differences in infestation found in our study were likely due to maternal oviposition choice. Fruit harvested from field efficacy trials were completely intact, so resulting offspring were eggs or neonate larvae when they were returned to the lab for rearing. Therefore, exposure to the repellent during development was minimal. The insecticidal properties of octenol have not been investigated for D. suzukii; however, larval development of D. melanogaster is negatively affected by volatile octenol (Inamdar et al. 2010, Yin et al. 2015). Additionally, Yin et al. (2015) described the S-enantiomer of 1-octen-3-ol as marginally more deleterious than the R-enantiomer, a common component of mushroom volatile profiles (Combet et al. 2006). As a commonly occurring volatile organic compound, the "safer" enantiomer may be something regularly encountered by insects in very low concentrations and more likely to be ignored. While the racemic blend used in our study minimizes the material cost of the repellent, further study is warranted, as the S-enantiomer may provide a more robust signal to egg-laying females of poor oviposition substrate quality.

Several chemicals have been reported as potential repellents or oviposition deterrents for the control of D. suzukii (Pham and Ray 2015, Wallingford et al. 2015, Erland et al. 2015, Renkema et al. 2016); however, a major barrier to the use of repellents in agricultural system is maintaining relatively high concentrations of a volatile chemical over long periods of time. We considered evaporation rates above $10 \mathrm{mg} / \mathrm{h}$ to be biologically relevant, and that any D. sukukii encountering concentrations such as these in the field would be induced to search for food sources or oviposition sites elsewhere. While preliminary testing of weight loss from repellent SPLAT material estimated evaporation rates that exceeded this target for longer than a week (data not shown), we hypothesize that release rates were dramatically altered due to our choices in experimental deployment. While the target release rate was exceeded on the day of deployment, estimated release rates fell well below our target after $1 \mathrm{~d}$ of aging (Fig. 1). It is likely that the repellent alcohol was wicked away by the paper from the SPLAT material, thereby negatively altering the longevity of the material. Additionally, our method of estimating evaporation rates does not include variations due to changes in moisture or fluctuating temperature. This could be of particular importance, as behaviors associated with mating and oviposition occur in the crepuscular hours when conditions are often cooler (Lin et al. 2014, Ferguson et al. 2015).

The odor deployed here, 1-octen-3-ol, is generally considered an aversive odor for drosophilids (Knaden et al. 2012) and likely acts as a feedback inhibition signal for fungal mycelial growth (Chitarra et al. 2004, 2005; Yin et al. 2015). However, both positive and negative behavioral or physiological changes have been reported in response to volatile 1-octen-3-ol for a wide range of taxa, and these responses are not necessarily consistent within groups. Several hematophagous arthropods are attracted to 1-octen-3-ol, as this compound is a component of mammalian odor profiles and a likely product of the microbial organisms living on their host's skin (Hall 
et al. 1984, Cork and Park 1996, Young et al. 2015). However, the southern house mosquito (Culex quinquefasciatus Say), which relies on bloodmeals from a broader host range (mammals and birds), is repelled by 1-octeno-3-ol (Xu et al. 2015). Behavioral responses to octenol are often attributed to that organism's relationship with the fungal organisms producing the odor and is found to be attractive to fungivorous insects (Combet et al. 2006). Several studies have identified 1-octen-3-ol as a component of plant odor profiles, perhaps originating from epiphytic yeasts and fungi, which are attractive to pollinator wasps, predatory mites, and phytophagous dipterans, lepidopterans, and coleopterans (Ozawa et al. 2000, Schlyter et al. 2000, Nojima et al. 2003, Beck et al. 2012, Brodmann et al. 2012, Jayanthi et al. 2012, Bendera et al. 2015). While the valence of behavioral response to 1-octen-3-ol is unpredictable, detection of the odor by insects is common and the neural pathways involved in detection are likely to be highly conserved (Inamdar et al. 2010).

The high release rate of repellent in the first days of deployment offered good conditions for evaluating its nontarget effects in the field. Hymenopteran behavior is particularly critical in raspberry systems, as bees are the primary pollinators (Wilmer et al. 1994). Our observations showed that pollinators were not avoiding plots treated with the repellent. Surprisingly, more visits by bumble bees were observed in repellent plots than control plots (Fig. 2). This increase in number of visits could indicate an attractant effect; however, this is unclear, as the number of visits to flowers was recorded rather than the number of insects visiting plots. Alternatively, detection of the repellent may have resulted in decreased duration of flower visits which would therefore lead to increases in the number of flowers visited. Further study is warranted to explore the role of volatile octenol on pollinator behavior, as an increase in number of visits may indicate an unpleasant effect on bumble bees.

Biological control is not always compatible with the regular foliar insecticide applications, and there are only a few products registered for use on fruit crops that are safe for important natural enemies (Desneux et al. 2007, Roubous et al. 2014, Biondi et al. 2016). There are several capable predators and pathogens that attack D. suzukii (Cuthbertson et al. 2014, Woltz et al. 2015, Renkema 2015). Parasitism by native wasp species in North America and Europe is low for this invasive pest (Chabert et al. 2012; Gabarra et al. 2015; Miller et al. 2015; Stacconi et al. 2013, 2015); there have been several parasitoids of D. suzukii described from its native range and classical biological control is a possibility (Mitsui and Kimura 2010, Kasuya et al. 2013, Nomano et al. 2015, Wang et al. 2016). Pest managers attempting to avoid secondary pest outbreaks due to the loss of natural enemies or wishing to minimize impacts on introduced D. suzuki predators in the future, may benefit by using a repellent. While further investigation may be necessary to evaluate the effect of a repellents on the hymenopterans attacking D. suzukii, we found no difference in the number of wasps trapped in repellent-treated versus control plots. This implies that hymenopterans did not avoid areas where the octenol was deployed and their visual attraction to yellow sticky traps was not impaired. However, further investigation may be warranted for deployment strategies that maintain high concentrations of repellent over longer periods of time.

In conclusion, despite issues with deployment, 1-octen-3-ol has been demonstrated to reduce infestation by field populations of D. suzukii in red raspberry. With improvements to deployment, a repellent could contribute to an integrated pest management program for control of D. suzukii, but nontarget effects should always be considered.

\section{Acknowledgments}

We would like to thank Karen Wentworth and Steve Hesler for their assistance with field experiments. Thank you to Kavita Sharma and Jonathan Rico with their assistance with SPLAT formulations. Thank you to our participating grower for the use of the commercial raspberry site. This research was supported in part by funding from Cornell University's New York State Agricultural Experiment Station federal formula funds, project 2012-13-195, New York State Department of Agriculture \& Markets Specialty Crops Block Grant C200783, New York State Berry Growers Association, and New York State Farm Viability Initiative FOC 14001 to G.L.

\section{References}

Asplen, M. K., G. Anfora, A. Biondi, D. Choi, D. Chu, K. M. Daane, P. Gibert, A. P. Gutierrez, K. A. Hoelmer, W. D. Hutchison, et al. 2015. Invasion biology of spotted wing Drosophila (Drosophila suzukii): A global perspective and future priorities. J. Pest Sci. 88: 469-494. DOI 10.1007/ s10340-015-0681-z.

Atallah, J., L. Teixeira, R. Salazar, G. Zaragoza, and A. Kopp. 2014. The making of a pest: The evolution of a fruit-penetrating ovipositor in Drosophila suzukii and related species. Proc. R. Soc. B 281: 20132840.

Beck, J. J., B. S. Higbee, D. M. Light, W. A. Gee, G. B. Merrill, and J. M. Hayashi. 2015. Hull split and damaged almond volatiles attract male and female navel orangeworms. J. Agric. Food Chem. 60: 8090-8096.

Beers, E. H., R. A. Van Steenwyk, P. W. Shearer, W. W. Coates, and J. A. Grant. 2011. Developing Drosophila suzukii management programs for sweet cherry in the western United States. Pest Manag. Sci. 67: 1386-1395.

Bendera, M., S. Ekesi, M. Ndug'u, R. Srinivasan, and B. Torto. 2015. A major host plant volatile, 1-octen-3-ol, contributes to mating in the legume pod borer, Maruca vitrata (Fabricius) (Lepidoptera: Crambidae). Naturwissenschaften 102: 47. DOI 10.1007/s00114-015-1297-0.

Biondi, A., V. Mommaert, G. Smagghe, E. Vinuela, L. Zappalà, and N. Desneux. 2016. The non-target impact of spinosyns on beneficial arthropods. Pest Manag. Sci. 68: 1523-1536.

Brodmann, J., D. Emer, and M. Ayasse. 2012. Pollinator attraction of the wasp-flower Scrophularia umbrosa (Scrophulariaceae). Plant Biol. 14: 500-505.

Chabert, S., R. Allemand, M. Poyet, P. Eslin, and P. Gibert. 2012. Ability of European parasitoids (Hymenoptera) to control a new invasive Asiatic pest, Drosophila suzukii. Biol. Control 63: 40-47.

Chitarra, G. S., T. Abee, F. M. Rombouts, M. A. Posthumus, and J. Dijksterhuis. 2004. Germination of Penicillium paneum conidia is regulation by 1-octen-3-ol, a volatile self-inhibitor. Appl. Environ. Microbiol. 70: 2823-2829.

Chitarra, G., T. Abee, F. M. Rombouts, and J. Dijksterhuis. 2005. 1-Octen-3ol inhibits conidia germination of Penicillium paneum despite of mild effects on membrane permeability, respiration, intracellular $\mathrm{pH}$, and changes the protein composition. FEMS Microbiol. Ecol. 54: 67-75.

Combet, E., J. Henderson, D. C. Eastwood, and K. S. Burton. 2006. Eight-carbon volatiles in mushrooms and fungi: Properties, analysis, and biosynthesis. Mycoscience 47: 317-326.

Cook, S. M., Z. R. Khan, and J. A. Pickett. 2007. The use of push-pull strategies in integrated pest management. Ann. Rev. Entomol. 52: 375-400.

Cork, A., and K. C. Park. 1996. Identification of electrophysiologically-active compounds for the malaria mosquito. Med. Vet. Entomol. 10: 269-276.

Crane, E., and P. Walker. 1984. Pollination directory for world crops. International Bee Research Association, London, p. 183.

Cuthbertson, A.G.S., L. S. Blackburn, and N. Audsley. 2014. Efficacy of commercially available invertebrate predators against Drosophila suzukii. Insects 5: 952-960.

Desneux, N., A. Decourtyre, and J. M. Delpuech. 2007. The sublethal effects of pesticides on beneficial arthropods. Ann. Rev. Entomol. 52: 81-106.

Diepenbrock, L. M., D. O. Rosensteel, J. A. Hardin, A. A. Sial, and H. J. Burrack. 2016. Season-long programs for control of Drosophila suzukii in southeastern U.S. blueberries. Crop Prot. 81: 76-84.

Erland, A. E., M. R. Rheault, and S. S. Mahmoud. 2015. Insecticidal and oviposition deterrent effects of essential oils and their constituents against the 
invasive pest Drosophila suzukii (Matsumura) (Diptera: Drosophilidae). Crop Prot. 78: 20-26.

Ferguson, C.T.J., T. L. O’Neill, N. Audsley, and R. E. Isaac. 2015. The sexually dimorphic behaviour of adult Drosophila suzukii: elevated female locomotor activity and loss of siesta is a postmating response. J. Exp. Biol. 218: 3855-3861.

Gabarra, R., J. Riudavets, G. A. Rodríguez, J. Pujade-Villar, and J. Arnó. 2015. Prospects for the biological control of Drosophila suzukii. BioControl 60: 331-339.

Hall, D. R., P. S. Beevor, A. Cork, B. F. Nesbitt, and G. A. Vale. 1984. 1-Octen-3-ol. A potent olfactory stimulant and attractant for tsetse isolated from cattle odours. Insect Sci. Appl. 5: 335-339.

Hampton, E., C. Koski, O. Barsoian, H. Faubert, R. S. Cowles, and S. R. Alm. 2014. Use of early ripening cultivars to avoid infestation and mass trapping to manage Drosophila suzukii (Diptera: Drosophilidae) in Vaccinium corymbosum (Ericales: Ericaceae). J. Econ. Entomol. 107: 1849-1857.

Haye, T., P. Girod, A.G.S. Cuthbertson, X. G. Wang, K. M. Daane, K. A. Hoelmer, C. Baroffio, J. P. Zhang, and N. Desneux. 2016. Current SWD IPM tactics and their practical implementation in fruit crops across different regions around the world. J. Pest Sci. 1-9. DOI 10.1007/s10340-0160737-8

Inamdar, A. A., P. Masurekar, and J. W. Bennett. 2010. Neurotoxicity of fungal volatile organic compounds in Drosophila melanogaster. Toxicol. Sci. 117: 418-426.

Jayanthi, P. D., C. M. Woodcock, J. Caulfield, M. A. Birkett, and T. J. Bruce. 2012. Isolation and identification of host cues from mango, Mangifera ind$i c a$, that attract gravid female oriental fruit fly, Bactrocera dorsalis. J. Chem. Ecol. 38: 361-369.

Kasuya, N., H. Mitsui, S. Ideo, M. Watada, and M. T. Kimura. 2013. Ecological, morphological and molecular studies on Ganaspis individuals (Hymenoptera: Figitidae) attaching Drosophila suzukii (Diptera: Drosophilidae). Appl. Entomol. Zool. 48: 87-92.

Klick, J., W. Q. Yang, V. M. Walton, D. T. Dalton, J. R. Hagler, A. J. Dreves, L. C. Lee, and D. J. Bruck. 2016. Distribution and activity of Drosophila suzukii in cultivated raspberry and surrounding vegetation. J. Appl. Entomol. 140: 37-46.

Knaden, M., A. Strutz, J. Ahsan, S. Sachse, and B. S. Hansson. 2012. Spatial representation of odorant valence in an insect brain. Cell Rep. 1: 392-399.

Lee, J. C. 2009. Parasitic Hymenoptera quick guide. (http://www.ars.usda. gov/SP2UserFiles/person/41853/PDF/articlesandinfo/Hymenoptera-Guide. pdf) (accessed December 2015).

Lee, J. C., A. J. Dreves, A. M. Cave, S. Kawai, R. Isaacs, J. C. Miller, S. Van Timmeren, D. J. Bruck. 2015. Infestation of wild and ornamental noncrop fruits by Drosophila suzukii (Diptera: Drosophilidae). Ann. Entomol. Soc. Am. 108: 117-129.

Lin, Q., Y. Zhai, C. Zhou, L. Li, Q. Zhuang, X. Zhang, F. G. Zalom, and Y. Yu. 2014. Behavioral rhythms of Drosophila suzukii and Drosophila melanogaster. Fla. Entomol. 97: 1424-1433.

Lupi, E., C. Hatz, and P. Schlagenhauf. 2013. The efficacy of repellents against Aedes, Anopheles, Culex and Ixodes spp. - A literature review. Travel Med. Infect. Dis. 11: 374-411.

Ozawa, R., T. Shimoda, M. Kawaguchi, G. Arimura, J. Horiuchi, T. Nishioka, and J. Takabayashi. 2000. Lotus japonicus infested with herbivorous mites emits volatile compounds that attract predatory mites. J. Plant Res. 113: 427-433.

Nomano, F. Y., H. Mitsui, and M. T. Kimura. 2015. Capacity of Japanese Asobara species (Hymenoptera; Braconidae) to parasitize a fruit pest Drosophila suzukii (Diptera; Drosophilidae). J. Appl. Entomol. 139: 105-113.

Miller, B., G. Anfora, M. Buffington, K. M. Daane, D. T. Dalton, K. Hoelmer, M.V.R. Stacconi, A. Grassi, C. Ioriatti, A. Loni, et al. 2015. Seasonal occurrence of resident parasitoids associated with Drosophila suzukii in two small fruit production regions of Italy and the USA. Bull. Insectol. 68: $255-263$.
Mitsui, H., and M. T. Kimura. 2010. Distribution, abundance and host association of two parasitoid species attacking frugivorous drosophilid larvae in central Japan. Eur. J. Entomol. 107: 535-540.

Nojima, A., C. Linn Jr., and W. Roelofs. 2003. Identification of host fruit volatiles from flowering dogwood (Cornus florida) attractive to dogwood-origin Rhagoletis pomonella flies. J. Chem. Ecol. 29: 2347-2357.

Pham, C. K., and A. Ray. 2015. Conservation of olfactory avoidance in Drosophila species and identification of repellents for Drosophila suzukii. Sci. Rep. 5: 11527.

Pimentel, D. 1995. Amounts of pesticides reaching target pests: Environmental impacts and ethics. J. Agric. Environ. Ethics 8: 17-29.

Renkema, J. M., Z. Telfer, T. Gariepy, and R. H. Hallett. 2015. Dalotia coriaria as a predator of Drosophila suzukii: Functional responses, reduced fruit infestation and molecular diagnostics. Biol. Control 89: 1-10.

Renkema, J. M., D. Wright, R. Buitenhuis, and R. H. Hallett. 2016. Plant essential oils and potassium metabisulfite as repellents for Drosophila suzukii (Diptera: Drosophilidae). Sci. Rep. 6: 21432.

Roubous, C. R., C. Rodriguez-Saona, R. Holdcraft, K. S. Mason, and R. Isaacs. 2014. Relative toxicity and residual activity of insecticides used in blueberry pest management: Mortality of natural enemies. J. Econ. Entomol. 107: 277-285.

Schlyter, F., Q. Zhang, P. Anderson, J. A. Byers, L. J. Wadhams, J. Löfqvist, and G. Birgersson. 2000. Electrophysiological and behavioural responses of Tomicus piniperda and Tomicus minor (Coleoptera: Scolytidae) to non-host leaf and bark volatiles. Can. Entomol. 132: 965-981.

Stacconi, M.V.R., A. Grassi, D. T. Dalton, B. Miller, M. Ouantar, A. Loni, C. Ioriatti, V. M. Walton, and G. Anfora. 2013. First field records of Pachycrepoideus vindemiae as a parasitoid of Drosophila suzukii in European and Oregon small fruit production areas. Entomologia 1: e3.

Stacconi, M.V.R., M. Buffington, K. M. Daane, D. T. Dalton, A. Grassi, G. Kaçar, B. Miller, J. C. Miller, N. Baser, C. Ioriatti, et al. 2015. Host stage preference, efficacy and fecundity of parasitoids attacking Drosophila suzukii in newly invaded areas. Biol. Control 84: 28-35.

Van Timmerren, S., and R. Isaacs. 2013. Control of spotted wing drosophila, Drosophila suzukii, by specific insecticides and by conventional and organic crop protection programs. Crop Prot. 54: 126-133.

Wallingford, A. K., S. P. Hesler, D. H. Cha, and G. M. Loeb. 2015. Behavioral response of spotted-wing drosophila, Drosophila suzukii Matsumura, to aversive odors and a potential oviposition deterrent in the field. Pest Manag. Sci. 72: 701-706. DOI 10.1002/ps.4040

Walsh, D. B., M. P. Bolda, R. E. Goodhow, A. J. Dreves, J. Lee, D. V. Bruck, V. M. Walton, S. D. O'Neal, and F. G. Zalom. 2011. Drosophila suzukii (Diptera: Drosophilidae): invasive pest of ripening soft fruit expanding its geographic range and damage potential. J. Integ. Pest Manag. 106: 289-295.

Wilmer, P. G., A.A.M. Bataw, and J. P. Hughes. 1994. The superiority of bumblebees to honeybees as pollinators: Insect visits to raspberry flowers. Ecol. Entomol. 19: 271-284.

Wang, X. G., G. Kacar, A. Biondi, and K. M. Daane. 2016. Foraging efficiency and outcomes of interactions of two pupal parasitoids attacking the invasive spotted wing drosophila. Biol. Control 96: 64-71.

Woltz, J. M., K. M. Donahue, D. J. Bruck, and J. C. Lee. 2014. Efficacy of commercially available predators, nematodes and fungal entomopathogens for augmentative control of Drosophila suzukii. J. Appl. Entomol. 139: 759-770

Yin, G., S. Padhi, S. Lee, R. Hung, G. Zhao, and J. W. Bennett. 2015. Effects of three volatile oxylipins on colony development in two species of fungi and on Drosophila larval metamorphosis. Curr. Microbiol. 71: 347-356. DOI 10.1007/s00284-015-0864-0

Young, R. M., N. D. Burkett-Cadena, T. W. McGaha Jr., M. A. RodriguezPerez, L. D. Toé, M. A. Adeleke, M. Sanfo, T. Soungalo, C. R. Katholi, R. Noblet, et al. 2015. Identification of human semiochemicals attractive to the major vectors of onchocerciasis. PLoS Negl. Trop. Dis. 9: e3450.

Xu, P., F. Zhu, G. K. Buss, and W. S. Leal. 2015. 1-Octen-3-ol - the attractant that repels. [version 1; referees: 4 approved] F1000Research 4:156. 